\title{
On Local Solutions of the Initial Value Problem for the Vlasov-Maxwell Equation
}

\section{Kiyoshi Asano}

Institute of Mathematics, Yoshida College, Kyoto University, Kyoto 606, Japan

\begin{abstract}
The initial value problem of the Vlasov-Maxwell equation has a unique solution in a time interval $[0, T]$ for each initial data in some function space. $T$ is estimated by the size of the initial data. The solution is classical, if the initial data is smooth.
\end{abstract}

\section{Introduction}

The density distribution of the charged gas particles changes under the rule described as the Vlasov-Maxwell equation. In this paper we prove that the initial value problem for the Vlasov-Maxwell equation has a unique local (in time) solution for each initial data in a slightly wide class of functions.

Let $f_{i}=f_{i}(t, x, v)$ be the density distribution of the charged gas particles of the type $i(1 \leqq i \leqq N)$ at the time $t \geqq 0$ and the point $x \in R^{3}$ with the velocity $v \in R^{3}$. The Vlasov-Maxwell equation for $\left\{f_{i}\right\}$ is described in the following form:

$$
\begin{aligned}
& \frac{\partial}{\partial t} f_{i}+v \cdot \nabla_{x} f_{i}+\frac{q_{i}}{m_{i}}\left(E+\frac{v}{c} \times B\right) \cdot \nabla_{v} f_{i}=0 \quad(1 \leqq i \leqq N), \\
& \left.f_{i}\right|_{t=0}=f_{i, 0}(x, v), \\
& \frac{\partial}{\partial t} E-c \nabla_{x} \times B=-4 \pi \sum_{\kappa}^{N} q_{i} \int v f_{i}(t, x, v) d v, \\
& \frac{\partial}{\partial t} B+c \nabla_{x} \times E=0 . \\
& \left.E\right|_{t=0}=E_{0}(x),\left.\quad B\right|_{t=0}=B_{0}(x)
\end{aligned}
$$

where $E$ and $B$ denote the electric and magnetic fields generated by the distributions $f_{i}, m_{i}$ the mass and $q_{i}$ the charge of the single particle of the $i$-species. The parameter $c \geqq 1$ denotes the light velocity. The notations ' and $\times$ denote the scalar and vector products in $\left.R^{3} ; \nabla_{x}={ }^{t} \partial / \partial x_{1}, \partial / \partial x_{2}, \partial / \partial x_{3}\right)$ and $\nabla_{v}={ }^{t}\left(\partial / \partial v_{1}, \partial / \partial v_{2}, \partial / \partial v_{3}\right)$. Sometimes we use the notations $\langle$,$\rangle and ||$ to denote the scalar product and the norm in $R^{n}$. 
From the first equation of (1.2) we obtain

$$
\frac{\partial}{\partial t} \nabla_{x} \cdot E=-4 \pi \Sigma q_{i} \int v \cdot \nabla_{x} f_{i} d v
$$

It is easy to see that (1.1)-(1.3) imply

$$
\nabla_{x} \cdot E-\nabla_{x} \cdot E_{0}(x)=4 \pi \Sigma q_{i} \int f_{i} d v-4 \pi \Sigma q_{i} \int f_{i, 0} d v, \quad \nabla_{x} \cdot B=\nabla_{x} \cdot B_{0} .
$$

Hence, to solve the above system of equations we have to put the compatit conditions,

$$
\nabla_{x} \cdot E_{0}=4 \pi \sum_{i=0}^{N} q_{i} \int_{R^{3}} f_{i, 0}(x, v) d v, \quad \nabla_{x} \cdot B_{0}=0
$$

If we assume

$$
B(t, x) \equiv B_{0}(x)
$$

then the second equation of (1.2) reduces to

$$
\nabla_{x} \times E(t, x)=0 .
$$

Combining (1.4) and (1.5) with (1.7), we have

$$
E(t, x)=\nabla_{x} \Sigma q_{i} \iint \frac{1}{|x-y|} f(t, y, v) d y d v .
$$

The system of Eqs. (1.1) and (1.8) is called the Vlasov-Poisson equation. Many authors have considered this type of equation. For example, see Arsen'ev [1], Bardos-Degond [2], Batt [3], Iordanskii [6] and Ukai-Okabe [8].

On the other hand the Vlasov-Maxwell equation has been studied by rather few authors. See Cooper-Klimas [4], Duniec [5], Neunzert-Petry [7] and Wollmann [9]. In the study of the Vlasov-Maxwell equation the main difficulty occurs from the term $\left(v / c \times \nabla_{x} B\right) \cdot \nabla_{v} f$, when we estimate $\nabla_{x}^{\alpha} f$. Wollmann [9] avoided this difficulty by assuming that the initial data $f_{0}(x, v)$ has compact support. To treat general $f_{0}$ we introduce a Banach scale $H_{\rho, \beta}^{l}$ (see (1.10) for the definition) characterized by the weight function $\exp (\rho|v|)$, and obtain an estimate of Cauchy-Kowalevski type (Lemma 2.4). If we assume that $f_{0}(x, v)=0$ for $|v| \geqq R$, then we can apply a simpler scheme and do not need such a Banach scale (Theorem 3.2).

With the notations defined below (see (1.10)-(1.12)) we state our main result.

Theorem 1.1. Let $f_{0, i} \in H_{\rho, \beta}^{l}(1 \leqq i \leqq N)$ and $\left(E_{0}, B_{0}\right) \in H^{l}$ with $l \geqq 3, \rho>0$ and $\beta \in R$. Then there exists a solution $\left(f_{1}, \ldots, f_{N}, E, B\right)$ of the initial value problem for the VlasovMaxwell equation (1.1)-(1.2) in a time interval [0,T], which satisfies the following:

$$
\begin{gathered}
f_{i} \in C_{\gamma / c}^{0}\left([0, T] ; H_{\rho, \beta}^{l}\right) \cap C_{\gamma / c}^{1}\left([0, T] ; H_{\rho, \beta-1}^{l-1}\right), 1 \leqq i \leqq N, \\
(E \cdot B) \in C^{0}\left([0, T] ; H^{l}\right) \cap C^{1}\left([0, T] ; H^{l-1}\right) .
\end{gathered}
$$

Here $T>0$ and $\gamma>0$ depend on $\left|f_{i, 0}\right|_{l, \rho, \beta}(1 \leqq i \leqq N),\left|E_{0}\right|_{l}$ and $\left|B_{0}\right|_{l}$, but not on $c \in[1, \infty)$. The solution is unique in $\bigcap_{j=0}^{1} C^{j}\left([0, T] ; H_{0, \beta-j}^{2-j}\right) \times C^{j}\left([0, T] ; H^{2-j}\right)$ with 
$\beta>5 / 2 . \quad$ Moreover $\quad\left(f_{1}(c, t, x, v), \ldots, f_{N}(c, t, x, v), \quad E(c, t, x), \quad B(c, t, x)\right) \in \bigcap_{j=0}^{l} M^{j}$ $\left([1, \infty): C_{\gamma}^{0}\left([0, T] ; H_{\rho, \beta-j}^{l-j}\right) \times C^{0}\left([0, T] ; H^{l-j}\right)\right)$. If $\left(E_{0}, B_{0}\right)$ satisfies $(1.5)$, then $(E, B)$ satisfies (1.4).

Remark. Our solution is classical, if $f_{0} \in C^{1}\left(R^{6}\right) \cap H_{\rho, \beta}^{3}$.

We introduce function spaces of measurable functions $H^{l}=H^{l}\left(R^{3}\right)$ and $H_{\rho, \beta}^{l}=H_{\rho, \beta}^{l}\left(R^{6}\right)$ with $l=0,1, \ldots, \rho \in R$ and $\beta \in R$ by

(i) $H^{l} h(x) \Leftrightarrow(\partial / \partial x)^{\alpha} h(x) \in L^{2}\left(R^{3}\right)$ for $|\alpha| \leqq l$.

The norm $|h|_{l}$ is defined by

$$
|h|_{l}^{2}=\sum_{|\alpha| \leqq l} \int_{R^{3}}\left|(\partial / \partial x)^{\alpha} h(x)\right|^{2} d x .
$$

(ii) $H_{\rho, \beta}^{l} \in f(x, v) \Leftrightarrow \phi_{\rho, \beta}(v)(\partial / \partial x)^{\alpha}(\partial / \partial v)^{\alpha^{\prime}} f(x, v) \in L^{2}\left(R^{6}\right)$ for $|\alpha|+\left|\alpha^{\prime}\right| \leqq l$ with $\phi_{\rho, \beta}(v)=e^{\rho|v|}(1+|v|)^{\beta}$.

The norm $|f|_{l, \rho, \beta}$ is defined by

$$
|f|_{l, \rho, \beta}^{2}=\sum_{|\alpha|+\left|\alpha^{\prime}\right| \leqq l}\left|\phi_{\rho, \beta}\left(\frac{\partial}{\partial x}\right)^{\alpha}\left(\frac{\partial}{\partial v}\right)^{\alpha^{\prime}} f\right|_{L^{2}\left(R^{6}\right)}^{2} .
$$

Remark. We can define $H^{l}$ and $H_{\rho, \beta}^{l}$ for fractional $l$ by the use of Fourier transformation or the interpolation theory. This remark is used in the proof of Lemma 2.6.

Throughout the paper we assume that $H^{l}$ is the set of vector valued (i.e. $R^{n}$ - or $C^{n}$ valued) functions on $R^{3}$ and $H_{\rho, \beta}^{l}$ is the set of scalar or vector valued functions according to the situation.

For a (closed) domain $\Omega \subset R^{n}$ and a Banach space $Y$ with the norm | $\left.\right|_{Y}$ (or more generally for a linear topological space $Y$ ) we denote by $C^{m}(\Omega ; Y)$ the space of $Y$-valued functions which are $m$ times continuously differentiable on $\Omega$ in the topology of $Y$. We also denote by $B^{m}(\Omega ; Y)$ the subspace of $h(x) \in C^{m}(\Omega ; Y)$ whose derivatives $(\partial / \partial x)^{\alpha} h(x),|\alpha| \leqq m$, are bounded on $\Omega$. If $Y$ is a $\operatorname{Banach}$ space, $B^{m}(\Omega ; Y)$ is also a Banach space with the norm

$$
\|h\|_{Y, m}=\|h\|_{m}=\sum_{|\alpha| \leqq m} \sup _{x \in \Omega}\left|\left(\frac{\partial}{\partial x}\right)^{\alpha} h(x)\right|_{Y} .
$$

We denote by $M^{0}(\Omega ; Y)$ (respectively $\left.M^{j}(\Omega ; Y)\right)$ the space of $Y$-valued (strongly) measurable and bounded functions on $\Omega$ (respectively the space of functions whose derivatives in the distribution sense up to order $j$ are in $\left.M^{0}(\Omega ; Y)\right) . M^{j}(\Omega ; Y)$ is a Banach space with the ess-sup norm \|\|$_{Y, j}=\|\|_{j}$.

Using these notations, we define the function spaces:

(i) $C^{0}\left([0, T] ; H^{l}\right)$ and $M^{0}\left([0, T] ; H^{l}\right)$ with the norm $|h|_{l, T}=\sup |h(t)|_{l}$.

(ii) $C_{\gamma}^{0}\left([0, T] ; H_{\rho, \beta}^{l}\right) \ni f \quad$ (respectively $\left.M_{\gamma}^{0}\left([0, T] ; H_{\rho, \beta}^{l}\right) \ni f\right) \stackrel{\substack{0 \leqq t \leqq T \\ \Leftrightarrow} \rho-\gamma t, \beta}{(v)(\partial / \partial x)^{\alpha}}$ $(\partial / \partial v)^{\alpha^{\prime}} f(t, x, v) \in C^{0}\left([0, T] ; L^{2}\left(R^{6}\right)\right)$ (respectively $\left.M^{0}\left([0, T] ; L^{2}\left(R^{6}\right)\right)\right)$.

The norm is defined by

$$
|f|_{l, \rho, \beta, \gamma, T}=\sup _{0 \leqq t \leqq T}|f(t)|_{l, \rho-\gamma t, \beta} .
$$


(iii) $C_{\gamma}^{1}\left([0, T]: H_{\rho, \beta}^{l}\right) \ni f \quad$ (respectively $\left.\quad M_{\gamma}^{1}\left([0, T] ; H_{\rho, \beta}^{l}\right) \ni f\right) \Leftrightarrow f \quad$ and $\quad d f / d t \in$ $C_{\gamma}^{0}\left([0, T] ; H_{\rho, \beta}^{l}\right)\left(\right.$ respectively $\left.M_{\gamma}^{0}\left([0, T] ; H_{\rho, \beta}^{l}\right)\right)$.

Remark. In the space of functions $f(t, x, v)$ defined on $[0, T] \times R^{6}$, we sometimes use the weight function $\phi_{\rho-\gamma|t-s|, \beta}(v)$ instead of $\phi_{\rho-\gamma t, \beta}(v)$. However there are no essential differences and no confusions will occur.

We use the notations $C^{m}\left(R^{n}\right)=C^{m}\left(R^{n} ; R^{k}\right.$ or $\left.C^{k}\right)$ and $B^{m}\left(R^{n}\right)=B^{m}\left(R^{n} ; R^{k}\right.$ or $\left.C^{k}\right)$. $\dot{B}^{m}\left(R^{n}\right)$ is the subspace of $h(x) \in B^{m}\left(R^{n}\right)$ such that $(\partial / \partial x)^{\alpha} h(x),|\alpha| \leqq m$, tends to zero uniformly as $|x| \rightarrow \infty$. For $0<\theta<1, B^{m+\theta}\left(R^{n}\right)$ (respectively $\dot{B}^{m+\theta}\left(R^{n}\right)$ ) is the subspace of functions of $B^{m}\left(R^{n}\right)$ (respectively $\dot{B}^{m}\left(R^{n}\right)$ ) whose $m^{\text {th }}$ derivatives are uniformly Hölder continuous with the exponent $\theta$. Their norms are denoted by \|\|$_{m}$ and \|\|$_{m+\theta}$ :

$$
\begin{aligned}
\|h\|_{m} & =\sum_{|\alpha| \leqq m} \sup _{x}\left|\left(\frac{\partial}{\partial x}\right)^{\alpha} h(x)\right|, \\
\|h\|_{m+\theta} & =\|h\|_{m-1}+\sum_{|\alpha|=m}\left\|\left(\frac{\partial}{\partial x}\right)^{\alpha} h\right\|_{\theta}, \\
\|h\|_{\theta} & =\|h\|_{0}+\sup _{x \neq y}|h(x)-h(y)| /|x-y|^{\theta} .
\end{aligned}
$$

We also use the notation $C_{0}^{m}\left(R^{n}\right)$ for the subspace of functions $f \in C^{m}\left(R^{n}\right)$ with a compact support. For a (closed) domain $\Omega$ of $R^{n}, C_{0}^{m}(\Omega)$ denotes the set of functions $f \in C_{0}^{m}\left(R^{n}\right)$ such that $\operatorname{supp} f \subset \Omega$.

Remark. After the completion of this work the author learned the work of P. Degond [10], in which he proved our Theorem 3.2. He also proved the asymptotic approach of the solution of the Vlasov-Maxwell equation to the solution of the Vlasov-Poisson equation as the light velocity $c$ tends to $\infty$. The same problem is studied in [11].

\section{The Linear Equation}

In this section we solve Eqs. (1.1)-(1.1) and (1.2)-(1.2) $)_{0}$ independently. First we treat

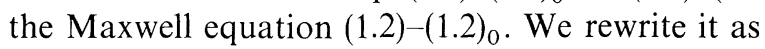

$$
\begin{aligned}
\frac{\partial}{\partial t}\left(\begin{array}{l}
E \\
B
\end{array}\right) & =\sum_{j=1}^{3} c A_{j} \frac{\partial}{\partial x_{j}}\left(\begin{array}{c}
E \\
B
\end{array}\right)+\left(\begin{array}{c}
F(t, x) \\
0
\end{array}\right), \\
\left.\left(\begin{array}{l}
E \\
B
\end{array}\right)\right|_{t=0} & =\left(\begin{array}{c}
E_{0} \\
B_{0}
\end{array}\right)
\end{aligned}
$$

where $A_{j}(1 \leqq j \leqq 3)$ and $F(t, x)$ are defined by

$$
\begin{aligned}
& A_{j}=\left(\begin{array}{rr}
0 & -\delta_{j} \\
\delta_{j} & 0
\end{array}\right), \quad \delta_{1}=\left(\begin{array}{rrr}
0 & 0 & 0 \\
0 & 0 & -1 \\
0 & 1 & 0
\end{array}\right), \\
& \delta_{2}=\left(\begin{array}{rrr}
0 & 0 & 1 \\
0 & 0 & 0 \\
-1 & 0 & 0
\end{array}\right), \quad \delta_{3}=\left(\begin{array}{rrr}
0 & -1 & 0 \\
1 & 0 & 0 \\
0 & 0 & 0
\end{array}\right),
\end{aligned}
$$




$$
F(t, x)=-4 \pi \sum_{i=1}^{n} q_{i} \int_{R^{3}} v f_{i}(t, x, v) d v .
$$

We assume with $l \geqq 1, \rho>0, \gamma \geqq 0, \rho-\gamma T \geqq \rho / 2>0$ and $\beta \in R$

$$
\begin{aligned}
& {[E B .0]^{l} \quad\left(E_{0}, B_{0}\right) \in H^{l},} \\
& {[F .1]_{\rho, \beta, \gamma, T}^{l} \quad f_{i} \in M_{\gamma}^{0}\left([0, T] ; H_{\rho, \beta}^{l}\right) \cap M_{\gamma}^{1}\left([0, T] ; H_{\rho, \beta-1}^{l-1}\right) .}
\end{aligned}
$$

Since Eq. (2.1) is a symmetric hyperbolic system, the operator $A=A_{1} \partial / \partial x_{1}+$ $A_{2} \partial / \partial x_{2}+A_{3} \partial / \partial x_{3}$ generates a group $e^{t A}$ of unitary operators in $H^{l}$. Hence Eq. $(2.1)-(2.1)_{0}$ has a solution described as

$$
\left(\begin{array}{c}
E(t) \\
B(t)
\end{array}\right)=e^{c t A}\left(\begin{array}{c}
E_{0} \\
B_{0}
\end{array}\right)+\int_{0}^{t} e^{c(t-s) A}\left(\begin{array}{c}
F(s, \cdot) \\
0
\end{array}\right) d s .
$$

It is easy to see that $F(t)$ satisfies

$$
\begin{gathered}
|F(t)|_{l} \leqq a(\rho, \beta) \sum_{\kappa^{=1}}^{N}\left|q_{i}\right|\left|f_{i}\right|_{l, \rho-\gamma t, \beta}, \\
a(\rho, \beta, \gamma, t) \leqq 4 \pi\left(\int_{R^{3}}|v|^{2} \phi_{\rho-\gamma t, \beta}^{-2}(v) d v\right)^{1 / 2} \leqq a(\rho / 2, \beta, 0,0) \equiv a(\rho, \beta) .
\end{gathered}
$$

From (2.4) we obtain the estimate for $(E(t), B(t))$, and we have

Lemma 2.1. Assume $[F .1]_{\rho, \beta, \gamma, T}^{l}$ and $[E B .0]^{l}$ with $l \geqq 1, \rho>0, \gamma>0, \rho-\gamma T \geqq \rho / 2>0$ and $\beta \in R$. Then Eq. (2.1)-(2.1) has a solution $(E(t, x), B(t, x))$ which is described by $(2.4)$ and satisfies

$$
\begin{aligned}
{[E B .1]_{T}^{l} \quad(E(t), B(t)) \in C^{0}(} & {\left.[0, T] ; H^{l}\right) \cap C^{1}\left([0, T] ; H^{l-1}\right), } \\
\left(|E(t)|_{l}^{2}+|B(t)|_{l}^{2}\right)^{1 / 2} \leqq & \left(\left|E_{0}\right|_{l}^{2}+\left|B_{0}\right|_{l}^{2}\right)^{1 / 2} \\
& +t a \sum_{i=1}^{N}\left|q_{i}\right|\left|f_{i}\right|_{l, \rho, \beta, \gamma, t}, \quad 0 \leqq t \leqq T,
\end{aligned}
$$

where $a=a(\rho, \beta)$ is defined in (2.5). The solution $(E(t), B(t))$ is unique in $C^{0}\left([0, T] ; H^{1}\right) \cap C^{1}\left([0, T] ; H^{0}\right)$.

Moreover, if $f_{i}(c, t, x, v)(1 \leqq i \leqq N)$ satisfy

$$
\left[F . \tilde{1}^{\prime}\right]_{\rho, \beta, \gamma, T}^{l} \quad f_{i} \in \bigcap_{j=0}^{l} M^{j}\left([1, \infty) ; M_{\gamma}^{0}\left([0, T] ; H_{\rho, \beta-j}^{l-j}\right)\right),
$$

then $(E(c, t, x), B(c, t, x))$ satisfies

$$
\left[E B . \tilde{1}^{\prime}\right]_{T}^{l} \quad(E, B) \in \bigcap_{j=0}^{l} M^{j}\left([1, \infty) ; C^{0}\left([0, T] ; H^{l-j}\right)\right) .
$$

When we solve the transport Eq. $(1.1)-(1.1)_{0}$, we can treat $N$-equations for $f_{1}, \ldots, f_{N}$ independently. Hence we have only to solve a single transport equation

$$
\begin{aligned}
L f & \equiv \frac{\partial}{\partial t} f+v \cdot \nabla_{x} f+\left(E+\frac{v}{c} \times B\right) \cdot \nabla_{v} f=k . \\
\left.f\right|_{t=s} & =f_{0}(x, v) .
\end{aligned}
$$


Here we have assumed without loss of generality that $q / m=1$. Consider the (backward) characteristic equation associated with (2.7):

$$
\left\{\begin{array} { l } 
{ \frac { d X } { d t } = - V , } \\
{ \frac { d V } { d t } = - E ( t , X ) - \frac { V } { c } \times B ( t , X ) , }
\end{array} \quad \left\{\begin{array}{l}
\left.X\right|_{t=s}=x \in R^{3} \\
\left.V\right|_{t=s}=v \in R^{3}
\end{array}\right.\right.
$$

and $(2.8)_{s}$

Assume

$$
\left[E B .1^{\prime}\right]_{T}^{l} \quad(E, B) \in C^{0}\left([0, T] ; H^{l}\right)
$$

with $l \geqq 3$ and $0<T<\infty$. The well known Sobolev theorem shows $H^{l}\left(R^{3}\right) \subset$ $\dot{B}^{l-3 / 2}\left(R^{3}\right)$ and $C^{0}\left([0, T] ; H^{l}\right) \subset C^{0}\left([0, T] ; \dot{B}^{l-3 / 2}\left(R^{3}\right)\right)$ with continuous inclusion, i.e,

$$
|h|_{l-3 / 2} \leqq b(l)|h|_{l}, \quad h \in H^{l}\left(\text { or } h \in C^{0}\left([0, T] ; H^{l}\right)\right)
$$

for $l \geqq 2$. Hence there is a unique solution $(X(t), V(t))$ of the initial value problem $(2.8)-(2.8)_{s}$, if $t$ is close to $s$. This solution is denoted as

$$
\begin{aligned}
& X(t)=X(t, s, x, v)=X(t, s, x, v ; E, B / c), \\
& V(t)=V(t, s, x, v)=V(t, s, x, v ; E, B / c) .
\end{aligned}
$$

Noting $V \cdot(V \times B)=0$, we get

$$
\left|\frac{d}{d t}\right| V(t)||=\frac{\left.\left|\frac{d}{d t}\right| V(t)\right|^{2} \mid}{2|V(t)|} \leqq\|E(t)\|_{0} \leqq\|E\|_{0, t} \leqq\|E\|_{0, T} .
$$

This gives

$$
\begin{aligned}
& |V(t, s, x, v)-v| \leqq|t-s|\|E\|_{0, t} \leqq|t-s|\|E\|_{0, T}, \\
& |X(t, s, x, v)-x| \leqq|t-s||v|+\frac{1}{2}|t-s|^{2}\|E\|_{0, T},
\end{aligned}
$$

for $0 \leqq t, s \leqq T$. Similarly we have

$$
|X(t, s, x, v)-x| \leqq|t-s||V|+\frac{1}{2}|t-s|^{2}\|E\|_{0, T}, \quad 0 \leqq s, t \leqq T,
$$

by solving the forward characteristic equation. The inequalities (2.12) and (2.13) show that the characteristic equation (2.8) has a global solution $(X(t, s, x, v), V(t, s, x, v))$ satisfying

$$
[S]_{T}^{l-2} \quad(X, V) \in C^{1}\left([0, T]^{2} ; C^{l-2}\left(R^{6}\right)\right) .
$$

We define a diffeomorphism $S(t, s)=S(t, s ; E, B / c)$ of $R^{6}$ by

$$
S(t, s)(x, v)=(X(t, s, x, v), V(t, s, x, v)), \quad(x, v) \in R^{6} .
$$

Since the vector field $(v, E(t, x)+v / c \times B(t, x))$ is of divergence free, $S(t, s)$ preserves the Lebesgue measure in $R^{6}$. Thus we have

Lemma 2.2. Assume $\left[E B .1^{\prime}\right]_{T}^{l}$ with $l \geqq 3$. Then there exists a unique solution $(X(t, s, x, v), V(t, s, x, v))$ of the (backward) characteristic Eq. (2.8)-(2.8), which satisfies $[S]_{T}^{l-2},(2.12),(2.13)$ and $(2.13)^{\prime}$. The $C^{l-2}$-diffeomorphism $S(t, s)$ of $R^{6}$ defined by $(2.14)$ 
preserves the Lebesgue measure in $R^{6}$ and satisfies

$$
S(t, s) S(s, r)=S(t, r) \text { and } S(t, t)=I=\text { identity, }
$$

$S(t, s)$ maps $\widetilde{B}_{R}$ (respectively $B_{R}$ ) into $\widetilde{B}_{\tilde{R}(T)}$ (respectively $B_{R(T)}$ )

and $R^{6} \backslash \widetilde{B}_{\tilde{R}(T)}$ (respectively $R^{6} \backslash B_{R(T)}$ ) into $R^{6} \backslash \widetilde{B}_{R}$ (respectively $R^{6} \backslash B_{R}$ ). (2.16)

Here $\widetilde{B}_{R}=\left\{(x, v) \in R^{6} ;|v| \leqq R\right\}, B_{R}=\left\{(x, v) ;|x|^{2}+|v|^{2} \leqq R^{2}\right\}, \widetilde{R}(T)=R+T\|E\|_{0, T}$ and $R(T)=C\left(T,\|E\|_{0, T}\right) R$ for $R \geqq 1$. The constant $C=C\left(T,\|E\|_{0, T}\right)$ depends only on $T$ and $\|E\|_{0, T}$ but not on $c \in[1, \infty)$.

Before solving Eq. (2.7)-(2.7) we give a result on the uniqueness of its solution.

Lemma 2.3. Let $(E, B) \in C^{0}\left([0, T] ; B^{0}\left(R^{3}\right)\right), k(t) \in M^{0}\left([0, T] ; H_{0, \beta}^{1}\right)$ and $f_{0} \in H_{0, \beta}^{1}$ with $\beta \in R$. Let $f$ and $g \in C^{0}\left([0, T] ; H_{0, \beta}^{1}\right) \cap C^{1}\left([0, T] ; H_{0, \beta-1}^{0}\right)$ be the solution of $(2.7)-(2.7)_{s}$. Then $f(t) \equiv g(t)$ for $0 \leqq t \leqq T$.

Proof. By the same calculation applied to prove (2.25), we have

$$
\begin{aligned}
\frac{d}{d t}|f-g|_{0,0, \beta-1}^{2} & =\frac{d}{d t}\left|\phi_{0, \beta-1}(f-g)\right|_{L^{2}\left(R^{6}\right)}^{2} \\
& \leqq 2|\beta-1|\|E\|_{0, T}|f-g|_{0,0, \beta-1}^{2}, \quad s \leqq t \leqq T .
\end{aligned}
$$

This proves the desired result.

Now we start to solve the transport Eq. (2.7)-(2.7). Assume $\left[E B \cdot 1^{\prime}\right]_{T}^{l}$ and $f_{0} \in H_{\rho, \beta}^{l}$ with $l \geqq 3, \rho>0$ and $\beta \in R$. Associated with the $C^{l-2}$-diffeomorphism $S(t, s)$, we define a linear operator $U(t, s)=U(t, s ; E, B / c)$ acting on $f_{0}$ :

$$
U(t, s) f_{0}(x, v)=U(t, s ; E, B / c) f_{0}(x, v)=f_{0}(S(t, s ; E, B / c)(x, v)) \text {. }
$$

Clearly $U(t, s) f_{0}$ is in $H_{\mathrm{loc}}^{l-2}\left(R^{6}\right)$ for $t \in[0, T]$ and satisfies Eq. $(2.7)-(2.7)_{s}$ with $k=0$. If we assume

$$
\left[F .2^{\prime}\right]_{\rho, \beta^{\prime}, \gamma, T}^{m} \quad k \in C_{\gamma}^{0}\left([0, T] ; H_{\rho, \beta^{\prime}}^{m}\right)
$$

with $m \geqq 1, \rho>0, \gamma \geqq 0$ and $\beta \in R$, then Eq. (2.7)-(2.7) sas a solution $f(t, x, v)$ described as

$$
f(t)=U(t, s) f_{0}+\int_{s}^{t} U(t, r) k(r) d r .
$$

To estimate $f(t)$ in $H_{\rho, \beta}^{l}$ we make temporary assumptions

$$
\begin{gathered}
(E, B) \in C^{0}\left([0, T] ; C^{l+1}\left(R^{3}\right)\right), \\
f_{0} \in C_{0}^{l+1}\left(R^{6}\right), \\
k \in C^{0}\left([0, T] ; C_{0}^{l+1}\left(R^{6}\right)\right), \\
\operatorname{supp} f_{0} \subset B_{R}, \operatorname{supp} k(t, \cdot) \subset B_{R}, R \geqq 1 .
\end{gathered}
$$

Then $S(t, s)$ is a $C^{l+1}$-diffeomorphism in $R^{6}$, and hence $f \in C^{0}([0, T]$; $\left.C_{0}^{l+1}\left(R^{6}\right)\right) \cap C^{1}\left([0, T] ; C_{0}^{l}\left(R^{6}\right)\right)$ and supp $f(t, \cdot) \subset B_{C R}$ by Lemma 2.2.

Denote by ||$_{0}$ and $($,$) the usual norm and scalar product in L^{2}\left(R^{6}\right)$. By applying 
the identity $v \cdot(v \times B)=0$ and partial integration we can easily show the equality

$$
\frac{1}{2} \frac{d}{d t}(g, g)=\operatorname{Re}(L g, g)
$$

for a nice function $g(t, x, v)$ and the differential operator $L$ of (2.7). Recall the weight function $\phi=\phi_{\rho-\gamma|t-s|, \beta}(v)$ (see (1.10) and (1.12)). We put

$$
g=g(t, x, v)=\phi f
$$

with $f$ of $(2.7) ; L f=k$. Since $v \cdot(v \times B)=0$, we have

$$
L g=(L \phi) f+\phi(L f)=\left\{-\gamma|v|+E\left(\rho-\gamma t+\beta(1+|v|)^{-1}\right) \frac{v}{|v|}\right\} \phi f+\phi k .
$$

If $\rho \geqq 0$ and $\gamma \geqq 0,(2.21)$ and (2.23) imply

$$
\begin{aligned}
\frac{1}{2} \frac{d}{d t}|\phi f|_{0}^{2} \leqq & -\left.\left.\gamma|| v\right|^{1 / 2} \phi f\right|_{0} ^{2}+(\rho+|\beta|)\|E(t, \cdot)\|_{0}|\phi f|_{0}^{2} \\
& +(\rho+|\beta|)|E(t, \cdot)|_{0}|\phi f|_{0}^{2}+|(\phi k, \phi f)|, \quad 0 \leqq s \leqq t \leqq T .
\end{aligned}
$$

We intend to apply (2.24) to $\partial^{\alpha} f=(\partial / \partial x)^{\alpha_{1}}(\partial / \partial v)^{\alpha_{2}} f, \alpha=\alpha_{1}+\alpha_{2}$. It follows from (2.7) that

$$
\begin{aligned}
L \partial^{\alpha} f & =\partial^{\alpha} k-G^{(\alpha)} \\
G^{(\alpha)} & =\left[\partial^{\alpha}, L\right] f=\left[\partial^{\alpha}, v \cdot \nabla_{x}\right] f+\left[\partial^{\alpha}, E \cdot \nabla_{x}\right] f+\left[\partial^{\alpha},\left(\frac{v}{c} \times B\right) \cdot \nabla_{v}\right] f .
\end{aligned}
$$

This gives

$$
\begin{aligned}
\frac{1}{2} \frac{d}{d t}\left|\phi \partial^{\alpha} f\right|_{0}^{2} \leqq & -\left.\left.\gamma|| v\right|^{1 / 2} \phi \partial^{\alpha} f\right|_{0} ^{2}+(\rho+|\beta|)\|E(t)\|_{0}\left|\phi \partial^{\alpha} f\right|_{0}^{2} \\
& +\left|\left(\phi \partial^{\alpha} k, \phi \partial^{\alpha} f\right)\right|+\left|\left(\phi G^{(\alpha)}, \phi \partial^{\alpha} f\right)\right|, \quad s \leqq t \leqq T .
\end{aligned}
$$

The estimate of $\left|\left(\phi G^{(\alpha)}, \phi \partial^{\alpha} f\right)\right|$ is given by the following

Lemma 2.4. Assume $\left[E B .1^{\prime}\right]_{T}^{l}$ and $[F .1]_{\rho, \beta, \gamma, T}^{l}$ with $l \geqq 3, \rho \geqq 0, \gamma \geqq 0$ and $\beta \in R$. Let $\partial^{\alpha}=(\partial / \partial x)^{\alpha_{1}}(\partial / \partial v)^{\alpha_{2}}=\partial_{x}^{\alpha_{1}} \partial_{v}^{\alpha_{2}}, \phi$ be the weight function and $c \in[1, \infty)$. Then for $1 \leqq$ $j \leqq l$, we have

$$
\begin{aligned}
\sum_{|\alpha| \leqq j}\left|\left(G(\alpha), \phi \partial^{\alpha} f\right)\right| \leqq & b(l)\left\{1+|\nabla E|_{l-1}+|B|_{l-1}\right\} \sum_{|\alpha| \leqq l}\left|\phi \partial^{\alpha} f\right|_{0}^{2} \\
& +c(l) c^{-1}|B|_{l} \sum_{|\alpha| \leqq l} \|\left.\left. v\right|^{1 / 2} \phi \partial^{\alpha} f\right|_{0} ^{2} .
\end{aligned}
$$

Here $b(l)$ and $c(l)$ are positive constants depending only on $l$.

Proof. By Leibniz formula we have

$$
\left[\partial^{\alpha}, v \cdot \nabla_{x}\right] f=\sum_{\sigma \leqq \alpha_{2},|\sigma|=1}\left(\begin{array}{c}
\alpha_{2} \\
\sigma
\end{array}\right) \partial_{v}^{\sigma} v \cdot \nabla_{x} \partial_{x}^{\alpha_{1}} \partial_{v}^{\alpha_{2}-\sigma} f .
$$


This implies

$$
\sum_{|\alpha| \leqq j}\left|\left(\phi\left[\partial^{\alpha}, v \cdot \nabla_{x}\right] f, \phi \partial^{\alpha} f\right)\right| \leqq b_{1}(l) \sum_{|\alpha| \leqq j}\left|\phi \partial^{\alpha} f\right|_{0}^{2} .
$$

Similarly we have

$$
\left[\partial^{\alpha}, E \cdot \nabla_{v}\right] f=\sum_{0<\sigma \leqq \alpha_{1}}\left(\begin{array}{c}
\alpha_{1} \\
\alpha
\end{array}\right) \partial_{x}^{\sigma} E \cdot \nabla_{v} \partial_{x}^{\alpha_{1}-\sigma} \partial_{v}^{\alpha_{2}} f
$$

Each term with $|\sigma| \leqq l-2$ is estimated as

$$
\begin{aligned}
& \left|\partial_{x}^{\sigma} E \cdot \nabla_{v} \partial_{x}^{\alpha_{1}-\sigma} \partial_{v}^{\alpha_{2}} f(\cdot, v)\right|_{L^{2}\left(R_{x}^{3}\right)} \\
& \quad \leqq\left\|\partial_{x}^{\sigma} E\right\|_{0}\left|\nabla_{v} \partial_{x}^{\alpha_{1}-\sigma} \partial_{v}^{\alpha_{2}} f(\cdot, v)\right|_{0}, \quad 1 \leqq|\sigma| \leqq l-2 .
\end{aligned}
$$

The remaining terms (if they exist) are estimated by using Sobolev theorem as follows.

$$
\begin{aligned}
& \left|\alpha_{x}{ }^{\sigma} E \cdot \nabla_{v} \partial_{x}^{\alpha_{1}-\sigma} \partial_{v}^{\alpha_{2}} f(\cdot, v)\right|_{L^{2}\left(R_{x}^{3}\right)} \\
& \quad \leqq\left|\partial_{x}{ }^{\sigma} E\right|_{L^{4}\left(R_{n}^{3}\right)}\left|\nabla_{v} \partial_{x}^{\alpha_{1}-\sigma} \partial_{v}^{\alpha_{2}} f(\cdot, v)\right|_{L^{4}\left(R_{x}^{3}\right)} \\
& \quad \leqq b_{1}\left|\partial_{x} E\right|_{L^{4}\left(R^{3}\right)}\left|\nabla_{v} \partial_{x}^{\alpha_{1}-\sigma} \partial_{v}^{\alpha_{2}} f(\cdot, v)\right|_{1} \quad \text { for }|\alpha|=l-1 \\
& \left|\partial_{x}{ }^{\sigma} E \cdot \nabla_{v} \partial_{x}^{\alpha_{1}-\sigma} \partial_{v}^{\alpha_{2}} f(\cdot, v)\right|_{L^{2}\left(R_{x}^{3}\right)} \leqq\left|\partial_{x}{ }^{\sigma} E\right|_{0}\left\|\nabla_{v} f(\cdot, v)\right\|_{0} \\
& \quad \leqq b_{2}\left|\partial_{x}{ }^{\sigma} E\right|_{0}\left|\nabla_{v} f(\cdot, v)\right|_{2} \text { for }|\sigma|=l
\end{aligned}
$$

Summing up the above results we obtain

$$
\sum_{|\alpha| \leqq j}\left|\left(\phi\left[\partial^{\alpha}, E \cdot \nabla_{v}\right] f, \phi \partial^{\alpha} f\right)\right| \leqq b_{2}(l)|\nabla E|_{l-1} \sum_{|\alpha| \leqq J}\left|\phi \partial^{\alpha} f\right|_{0}^{2}
$$

Finally we have,

$$
\begin{aligned}
& {\left[\partial^{\alpha},(v \times B) \cdot \nabla_{v}\right] f} \\
& \quad=\sum_{\sigma_{1} \leqq \alpha_{1}, 0<\sigma_{2} \leqq \alpha_{2}}\left(\begin{array}{c}
\alpha_{1} \\
\sigma_{1}
\end{array}\right)\left(\begin{array}{c}
\alpha_{2} \\
\sigma_{2}
\end{array}\right)\left(\partial_{v}^{\sigma_{2}} v \times \partial_{x}^{\sigma_{1}} B\right) \cdot \nabla_{v} \partial_{x}^{\alpha_{1}-\sigma_{1}} \partial_{v}^{\alpha_{2}-\sigma_{2}} f \\
& \quad+\sum_{0<\sigma \leqq \alpha_{1}}\left(\begin{array}{c}
\alpha_{1} \\
\sigma
\end{array}\right)\left(v \times \partial_{x}{ }^{\sigma} B\right) \cdot \nabla_{v} \partial_{x}^{\alpha_{1}-\sigma} \partial_{v}^{\alpha_{2}} f .
\end{aligned}
$$

Calculating similarly as above, we obtain

$$
\begin{aligned}
& \sum_{|\alpha| \leqq j}\left|\left(\phi\left[\partial^{\alpha},(v \times B) \cdot \nabla_{v}\right] f, \phi \partial^{\alpha} f\right)\right| \\
& \quad \leqq b_{3}(l)|B|_{l-1} \sum_{|\alpha| \leqq j}\left|\phi \partial^{\alpha} f\right|_{0}^{2}+c(l)|B|_{l} \sum_{|\alpha| \leqq j} \|\left.\left. v\right|^{1 / 2} \phi \partial^{\alpha} f\right|_{0} ^{2} .
\end{aligned}
$$

Summing up (2.29)-(2.31), we have the desired result (2.28).

If $\gamma$ satisfies

$$
\gamma \geqq c(l)|B|_{l, T}\left(\text { or } \gamma=\gamma(c) \geqq c(l)|B|_{l, T} / c\right)
$$


then (2.26) and (2.27) give

$$
\begin{aligned}
& \frac{d}{d t}|f(t)|_{j, \rho-\gamma t, \beta} \\
& \quad \leqq b\left\{|f(t)|_{j, \rho-\gamma t, \beta}+|k(t)|_{j, \rho-\gamma t, \beta}\right\}, \quad 1 \leqq j \leqq l \\
& b=b(l)\left\{1+(\rho+|\beta|+1)|E|_{l, T}+|B|_{l-1, T}\right\}
\end{aligned}
$$

This implies

$$
\begin{aligned}
|f(t)|_{j, \rho-\gamma t, \beta} \leqq & e^{b(t-s)}|f(s)|_{j, \rho-\gamma s, \beta} \\
& +\int_{s}^{t} e^{b(t-r)}|k(r)|_{j, \rho-\gamma r, \beta} d r, \quad 0 \leqq j \leqq l .
\end{aligned}
$$

Recalling Eq. (2.7) and the fact that $C_{0}^{l+1}\left(R^{6}\right)$ is dense in $H_{\rho, \beta}^{l}(\rho>0)$, we obtain under the condition (2.20)

$$
[F .2]_{\rho, \beta, \gamma, T}^{l} \quad f \in C_{\gamma}^{0}\left([0, T] ; H_{\rho, \beta}^{l}\right) \cap C_{\gamma}^{1}\left([0, T] ; H_{\rho, \beta-1}^{l-1}\right)
$$

with the weight function $\phi=\phi_{\rho-\gamma|t-s|, \beta}$.

We also note that under the assumption $\left[E B .1^{\prime}\right]_{T}^{l} f=U(t, s ; E, B / c) f_{0}$ satisfies $[F .2]_{\rho, \beta, \gamma, T}^{l-2}$ with the weight function $\phi=\phi_{\rho-\gamma|t-s|, \beta}$. This is shown by differentiating $U(t, s ; E, B / c) f_{0}$, recalling the condition $[S]_{T}^{l-2}$ and applying (2.34) with $j=l-2$. Thus $U(t, s ; E, B / c) f_{0}$ defined by (2.18) is the unique solution of Eq. (2.7)-(2.7) with $k=0$.

Summing up the above, we have

Lemma 2.5. Let $l \geqq 3, \rho>0, \beta \in R$ and $f_{0} \in H_{\rho, \beta}^{l}$.

(i) Assume $\left[E B .1^{\prime}\right]_{T}^{l}$ and choose $\gamma$ satisfying (2.32). Then $f=U(t, s ; E, B / c) f_{0}$ satisfies $[F .2]_{\rho, \beta, \gamma, T}^{l-2}\left(\right.$ with $\left.\phi_{\rho-\gamma|t-s|, \beta}\right)$ and is a unique solution of $(2.7)-(2.7)_{s}$ with $k=0$. If $k$ satisfies $[F .2]_{\rho, \beta, \gamma, T}^{m}\left(\right.$ respectively $\left.[F .1]_{\rho, \beta, \gamma, T}^{m}\right), 1 \leqq m \leqq l-2\left(\right.$ with $\left.\phi_{\rho-\gamma|t-s|, \beta}\right)$, then Eq. $(2.7)-(2.7)_{s}$ has a unique solution $f$ which is given by (2.19) and satisfies $[F .2]_{\rho, \beta, \gamma, T}^{m}$ (respectively $[F .1]_{\rho, \beta, \gamma, T}^{m}$ ).

(ii) Suppose $(E, B) \in C^{0}\left([0, T] ; C^{l+1}\left(R^{3}\right)\right)$ additionally. Then the linear operator $U(t, s ; E, B / c)$ is continuous from $H_{\rho, \beta}^{j}$ to $H_{\rho-\gamma|t-s|, \beta}^{j}, 0 \leqq j \leqq l$, and satisfies the estimate

$$
\left|U(t, s ; E, B / c) f_{0}\right|_{j, \rho-\gamma|t-s|, \beta} \leqq e^{b|t-s|}\left|f_{0}\right|_{j, \rho, \beta}
$$

for $c \in[1, \infty), t, s \in[0, T] 0 \leqq j \leqq l$ and $b$ in $(2.33)$. Moreover $U(t, s ; E, B / c)$ is continuous in $H_{\rho, \beta}^{0}$ and satisfies

$$
\left|U(t, s ; E, B / c) f_{0}\right|_{0, \rho, \beta} \leqq e^{|t-s|(\rho+|\beta|)|E|_{0, T}}\left|f_{0}\right|_{0, \rho, \beta} .
$$

(iii) Let $H^{j}\left(\widetilde{B}_{R}\right)$ be the closure of $C_{0}^{l+1}\left(\widetilde{B}_{R}\right)$ in $H^{j}\left(R^{6}\right)$. Let $f_{0} \in \stackrel{H}{ }^{j}\left(\widetilde{B}_{R}\right)$ with $0 \leqq j \leqq l$. Then, under the assumption of (ii), $U(t, s ; E, B / c) f_{0} \in C^{0}\left([0, T] ; H^{j}\left(\widetilde{\widetilde{B}}_{\tilde{R}(T)}\right) \cap\right.$ $C^{1}\left([0, T] ; H^{j-1}\left(B_{\tilde{R}(T)}\right)\right), \widetilde{R}(T)=R+|E|_{0, T}$, and satisfies (for $c \in[1, \infty)$ )

$$
\begin{aligned}
\left|U(t, s ; E, B / c) f_{0}\right|_{j, 0, \beta} & \leqq e^{\delta|t-s|}\left|f_{0}\right|_{j, 0, \beta}, \quad 0 \leqq j \leqq l, \\
\tilde{b} & =b+c(l)|B|_{l, T} R(T) .
\end{aligned}
$$


Remark. By virtue of the interpolation theorem, the estimates (2.35) and (2.37) hold for fractional $j \in[0, l]$.

Now we are at the final stage of this section. We prove the next

Lemma 2.6. Assume $\left[E B .1^{\prime}\right]_{T}^{l}$ and $f_{0} \in H_{\rho, \beta}^{l}$ with $l \geqq 3, \rho>0$ and $\beta \in R$. Let $\gamma$ satisfy (2.32) and $c \in[1, \infty)$. Then:

(i) $U(t, s ; E, B / c) f_{0}$ satisfies $[F .2]_{\rho, \beta, \gamma, T}^{l}$ (with the weight function $\left.\phi_{\rho-\gamma|t-s| \beta}\right),(2.35)$ and (2.36).

(ii) If supp $f_{0} \subset B_{R}$ (respectively $\operatorname{supp} f_{0} \subset B_{R}$ ) with $R \geqq 1$, then $U(t, s$; $E, B / c) f_{0}(x, v)=0 \quad$ for $\quad|v| \geqq R+|t-s|\|E\|_{0, T} \quad$ (respectively for $\quad|(x, v)| \geqq$ $\left.C\left(T,\|E\|_{0, T}\right) R\right)$ and satisfies (2.37).

(iii) Moreover, if $[E B . \tilde{1}]_{T}^{l}$ is assumed, then there holds

$$
[F .2]_{\rho, \beta, \gamma, T}^{l} \quad f \in \bigcap_{j=0}^{l-1} M^{j}\left([1, \infty) ; C_{\gamma}^{0}\left([0, T] ; H_{\rho, \beta-j}^{l-j}\right) \cap C_{\gamma}^{1}\left([0, T] ; H_{\rho, \beta-1-j}^{l-1-j}\right)\right)
$$

with $\phi_{\rho-\gamma|t-s|, \beta}$.

Proof. To prove (i) and (ii), we have only to show that we can remove the first condition of the temporary assumption (2.20). Since $H^{l} \cap C^{l+1}\left(R^{3}\right)$ is dense in $H^{l}$, we have an approximate sequence $\left(E_{n}, B_{n}\right) \in C^{0}\left([0, T] ; H^{l} \cap C^{l+1}\left(R^{3}\right)\right)$,

$$
\left|E_{n}-E\right|_{l, T}+\left|B_{n}-B\right|_{l, T} \rightarrow 0 \quad(n \rightarrow \infty)
$$

by using Friedrichs' mollifier. (This procedure is independent of $t \in[0, T]$ and $c \in[1, \infty)$.) We define the sequence of evolution operators $U_{n}(t, s)=U\left(t, s ; E_{n}, B_{n} / c\right)$ by $(2.18)$, replacing $(E, B)$ by $\left(E_{n}, B_{n}\right)$. Then $f_{n}(t, x, v)=U_{n}(t, s) f_{0}$ satisfies all the conditions and estimates described in Lemma 2.5 (with $k=0$ ) and also the equation

$$
\begin{gathered}
\frac{\partial}{\partial t} f_{n}+v \cdot \nabla_{x} f_{n}+\left(E_{n}+\frac{v}{c} \times B_{n}\right) \cdot \nabla_{v} f_{n}=0, \quad 0 \leqq t \leqq T . \\
\left.f_{n}\right|_{t=s}=f_{0}(x, v) .
\end{gathered}
$$

Since $\left|E_{n}\right|_{l, T}$ and $\left|B_{n}\right|_{l, T}$ can be assumed to be bounded by $|E|_{l, T}+\varepsilon$ and $|B|_{l, T}+\varepsilon$, respectively, with a small constant $\varepsilon>0$, we can choose $\gamma$ to satisfy the condition (2.32) uniformly for all $\left(E_{n}, B_{n}\right)$. All $U_{n}(t, s)$ satisfy the uniform estimates such as (2.35) and (2.37). An easy calculation shows

$$
\begin{aligned}
& \left\{\frac{\partial}{\partial t}+v \cdot \nabla_{x}+\left(E_{n}+\frac{v}{c} \times B_{n}\right) \cdot \nabla_{v}\right\}\left(f_{n}-f_{m}\right) \\
& =-\left(E_{n}-E_{m}\right) \cdot \nabla_{v} f_{m}-\frac{v}{c} \times\left(B_{n}-B_{m}\right) \cdot \nabla_{v} f_{m} \equiv k_{n, m} .
\end{aligned}
$$

From the uniqueness of $f_{n}-f_{m}$ (Lemma 2.3) it follows

$$
f_{n}(t)-f_{m}(t)=\int_{s}^{t} U_{n}(t, r) K_{n, m}(r) d r
$$

The estimate (2.34) established for $U_{n}(t, s)=U\left(t, s ; E_{n}, B_{n} / c\right)$ gives

$$
\left|f_{n}-f_{m}\right|_{0, \rho-\gamma|t-s|, \beta-1} \leqq \int_{s}^{t} e^{b_{0, n}|t-r|}\left|k_{n, m}\right|_{0, \rho-\gamma|t-c|, \beta-1} d r
$$




$$
\begin{aligned}
& \leqq|t-s| e^{b_{0}|t-s|}\left(\left|E_{n}-E_{m}\right|_{0, T}+\left|B_{n}-B_{m}\right|_{0, T}\right) \\
& \times \sup _{s \leqq r \leqq t}\left|\nabla f_{m}(r)\right|_{0, \rho-\gamma|t-r|, \beta},
\end{aligned}
$$

where $\quad b_{0, n}=(\rho+|\beta-1|)\left|E_{n}\right|_{0, T} \leqq(\rho+|\beta-1|)\left(|E|_{0, T}+b_{2} \varepsilon\right) \leqq b_{0} . \quad$ Since $\left|\nabla f_{m}(r)\right|_{0, \rho-\gamma|t-r|, \beta}=\left|\nabla U_{m}(t, r) f_{0}\right|_{0, \rho-\gamma|t-r|, \beta}$ is estimated by $(2.35)$, it is uniformly bounded in $m$ and $t, r \in[0, T]$ (and in $c \in[1, \infty)$ ). Hence $\left\{f_{n}(t)\right\}$ converges in $H_{\rho-\gamma|t-s|, \beta-1}^{0}$ uniformly in $t \in[0, T]$. Noting that (2.35) implies the uniform boundedness of $\left\{f_{n}(t)\right\}$ in $C_{\gamma}^{0}\left([0, T] ; H_{\rho, \beta}^{l}\right)$ and applying the interpolation theorem between $H_{\rho-\gamma \mid t-s, \beta-1}^{0}$ and $H_{\rho-\gamma|t-s|, \beta-1}^{l}$ and then between $H_{\rho-\gamma|t-s|, \beta-1}^{l-\delta}$ and $H_{\rho-\gamma|t-s|, \beta}^{l}$, we see that $\left\{f_{n}(t)\right\}$ converges in $H_{\rho-\gamma|t-s|, \beta-\delta}^{l-\delta}, 0<\delta \leqq 1$, uniformly in $t \in[0, T]$, and the limit $f(t) \in C_{\gamma}^{0}\left([0, T] ; H_{\rho, \beta-\delta}^{l-\delta}\right)$ (with $\left.\phi_{\rho,-\rho|t-s|, \beta-\delta}\right)$. Taking the equality (2.38) into account, we see also that $f \in C_{\gamma}^{1}\left([0, T] ; H_{\rho, \beta-1-\delta}^{l}(1-\delta)\right.$ and satisfies Eq. $(2.7)-(2.7)_{s}$ (with $k=0$ ).

If we construct $U(t, s)=U(t, s ; E, B / c)$ by $(2.18)$ from the original $(E, B)$, then $U(t, s) f_{0}$ satisfies $[F .2]_{\rho, \beta, \gamma, T}^{l-2}$ and Eq. (2.7)-(2.7) $($ with $k=0)$ (Lemma 2.5 (i)). By virtue of Lemma 2.3 we have

$$
\begin{aligned}
& U(t, s ; E, B / c) f_{0}=s-\lim U\left(t, s ; E_{n}, B_{n} / c\right) f_{0} \\
& \text { in } H_{\rho-\gamma|t-s|, \beta-\delta}^{l-\delta}, \quad 0<\delta \leqq 1, \quad t \in[0, T] \quad(\text { and } c \in[1, \infty)) .
\end{aligned}
$$

On the other hand, $\left\{f_{n}(t)\right\}$ is weakly pre-compact in $H_{\rho-\gamma|t-2|, \beta}^{l}$ and the only accumulation point is $f(t)$. Thus we see

$$
U(t, s ; E, B / c) f_{0}=w-\lim U\left(t, s ; E_{n}, B_{n} / c\right) f_{0} \quad \text { in } H_{\rho-\gamma|t-s|, \beta}^{l},
$$

and that the estimate $(2.35)$ holds for $U(t, s ; E, B / c) f_{0}$ constructed from the original $(E, B)$. Since the Hilbert space $H_{\rho-\gamma|t-s|, \beta}^{l}$ is separable, weak measurability of $f(t)$ in $[0, T]$ implies strong measurability.

If $f_{0} \in \dot{H}^{l}\left(\widetilde{B}_{R}\right)$, we can apply Lemma 2.5 (iii) to the sequence $\left\{f_{n}(t)=U_{n}(t, s) f_{0}\right\}$ in the same way as in the above argument. Then, we have

$$
\begin{aligned}
f(t) \equiv & U(t, s ; E, B / c) f_{0}=s-\lim f_{n}(t) \text { in } H^{l-\delta}\left(R^{6}\right), \quad 0<\delta \leqq 1, \\
f(t) \equiv & U(t, s ; E, B / c) f_{0}=w-\lim f_{n}(t) \text { in } H^{l}\left(R^{6}\right), \\
& \left|f_{n}(t)\right|_{l-\delta, 0,0} \leqq e^{\delta\left|t-t^{\prime}\right|}\left|f_{n}\left(t^{\prime}\right)\right|_{l-\delta, 0,0}, \quad 0 \leqq \delta \leqq 1
\end{aligned}
$$

for $t, t^{\prime} \in[0, T]$ and all $n$. Here $\tilde{b}$ can be chosen to be independent of $n$ (see (2.37)). Since $f \in C^{0}\left([0, T] ; H^{l-1}\left(R^{6}\right)\right)$ and $H^{l+1}\left(R^{6}\right)$ is dense in $H^{l}\left(R^{6}\right)$, it follows that $f(t)$ is weakly continuous in $H^{l}\left(R^{6}\right)$ on $[0, T]$.

We define the Fourier transform $\hat{h}(\xi, \eta)$ of $h(x, v)$ by

$$
\hat{h}(\xi, \eta)=(2 \pi)^{-3 / 2} \int_{R^{6}} e^{-i(x \cdot \xi+v \cdot \eta)} h(x, v) d x d v .
$$

If we define the norm $|h|_{j}$ of $h \in H^{j}\left(R^{6}\right)$ by

$$
|h|_{j}^{2}=\int_{R^{6}}\left(1+|\xi|^{2}+|\eta|^{2}\right)^{j}|\hat{h}(\xi, \eta)|^{2} d \xi d \eta, \quad 0 \leqq j \leqq l,
$$

the estimate (2.37), and hence (2.46), still hold for $j=0,1, \ldots, l$ with $\tilde{b}$ replaced by 
some appropriate constant (if necessary). We note that the norm $|h|_{j}$ defined by (2.47) is monotone increasing and continuous in $j \in[0, l]$ if $h \in H^{l}\left(R^{6}\right)$.

Let $n \rightarrow \infty$ and then $\delta \rightarrow 0$ in (2.46). Then (2.44) and (2.45) with the above remark give

$$
|f(t)|_{l} \leqq e^{b\left|t-t^{\prime}\right|}\left|f\left(t^{\prime}\right)\right|_{l}, \quad t, t^{\prime} \in[0, T] .
$$

This means that $|f(t)|_{l}$ is continuous on $[0, T]$. Thus we have proved that $f(t) \in C^{0}\left([0, T] ; H^{l}\left(R^{6}\right)\right)$. Since $\operatorname{supp} f(t) \subset \widetilde{B}_{\tilde{R}(T)}$ for $t \in[0, T]$, it follows that $f$ satisfies $\left[F .2^{\prime}\right]_{\rho, \beta, \gamma, T}^{l}$, and hence $[F .2]_{\rho, \beta, \gamma, T}^{l}$ for each $\rho \geqq 0, \beta \in R$ and $\gamma \geqq 0$. Since the union of $C_{0}^{l+1}\left(B_{R}\right)$ is dense in $H_{\rho, \beta}^{l}$, the proof of Lemma 2.6 (i) is completed.

The proof of (iii) is easily carried out.

\section{The Nonlinear Equation}

In this section we study the nonlinear Vlasov-Maxwell equation (1.1)-(1.2). For simplicity we study the following equation for the plasma of a single species:

$$
\begin{gathered}
\frac{\partial}{\partial t} f+v \cdot \nabla_{x} f+\left(E+\frac{v}{c} \times B\right) \cdot \nabla_{v} f=0, \quad t>0, \quad x \in R^{3}, \quad v \in R^{3}, \\
\left.f\right|_{t=0}=f_{0}(x, v), \\
\frac{\partial}{\partial t} E-c \nabla_{x} \times B=-4 \pi \int v f(t, x, v) d v, \\
\frac{\partial}{\partial t} B+c \nabla_{x} \times E=0, \quad t>0, \quad x \in R^{3}, \\
\left.E\right|_{t=0}=E_{0}(x),\left.\quad B\right|_{t=0}=B_{0}(x) .
\end{gathered}
$$

We prove the existence and uniqueness theorem for Eq. (3.1)-(3.2) (Theorem 3.1). However no essential differences occur in the proof of Theorem 1.1.

Theorem 3.1. Let $f_{0} \in H_{\rho, \beta}^{l}$ and $\left(E_{0}, B_{0}\right) \in H^{l}$ with $l \geqq 3, \rho>0$ and $\beta \in R$. Then there exists a solution $(f, E, B)$ of the initial value problem for the Vlasov-Maxwell equation $(3.1)-(3.1)_{0}$ and (3.2)-(3.2) $)_{0}$ in the time interval $[0, T]$ satisfying the following properties:

$$
\begin{gathered}
f \in C_{\gamma / c}^{0}\left([0, T] ; H_{\rho, \beta}^{l}\right) \cap C_{\gamma / c}^{1}\left([0, T] ; H_{\rho, \beta}^{l-1}\right) \equiv F_{\rho, \beta, \gamma / c, T}^{l}, \\
|f|_{l, \rho, \beta, \gamma / c, T} \leqq \widetilde{Y}_{0}=Z_{0}\left|f_{0}\right|_{l, \rho, \beta}, \\
(E, B) \in C^{0}\left([0, T] ; H^{l}\right) \cap C^{0}\left([0, T] ; H^{l-1}\right) \equiv E B_{T}^{l}, \\
|E|_{l, T}+|B|_{l, T} \leqq 2\left(\left|E_{0}\right|_{l}+\left|B_{0}\right|_{l}\right)+a T Y_{0}, \quad a=a(\rho, \beta) .
\end{gathered}
$$

Here $\gamma, T, Y_{0}$ and $Z_{0}$ depend on $l, \rho, \beta,\left|f_{0}\right|_{l, \rho, \beta},\left|E_{0}\right|_{l}$ and $\left|B_{0}\right|_{l}$ but not on $c \in[1, \infty)$, and are determined by (3.14), (3.15), (3.19) and the solvability conditions of (3.17) and (3.26). The solution $(F, E, B)$ is unique in

$$
\bigcap_{j=0}^{1} C^{j}\left([0, T] ; H_{0, \beta^{\prime}}^{2-j}\right) \times C^{j}\left([0, T] ; H^{2-j}\right) \text { with } \beta^{\prime}>5 / 2,
$$


and $f$ is described as $f(t)=U(t, 0 ; E, B) f_{0}$. Moreover

$$
(f, E, B) \in \bigcap_{j=0}^{l} M^{j}\left([1, \infty) ; C_{\gamma}^{0}\left([0, T] ; H_{\rho, \beta-j}^{l-j}\right) \times C^{0}\left([0, T] ; H^{l-j}\right)\right) .
$$

Proof. We define the sequence $\left(f_{n}(t), E_{n}(t), B_{n}(t)\right)$ by

$$
\begin{gathered}
\left(f_{0}(t), E_{0}(t), B_{0}(t)\right) \equiv\left(f_{0}(x, v), E_{0}(x), B_{0}(x)\right), \\
\left(\begin{array}{c}
E_{n}(t) \\
B_{n}(t)
\end{array}\right)=e^{c t A}\left(\begin{array}{c}
E_{0} \\
B_{0}
\end{array}\right)+\int_{0}^{t} e^{c(t-s) A}\left(\begin{array}{c}
F_{n-1}(s) \\
0
\end{array}\right) d s, \quad n \geqq 1, \\
F_{n}(t, x)=-4 \pi \int v f_{n}(t, x, v) d v, \quad n \geqq 0, \\
f_{n}(t)=U\left(t, 0 ; E_{n}, B_{n} / c\right) f_{0}, \quad n \geqq 1 .
\end{gathered}
$$

If we assume that

$$
\begin{gathered}
\left(f_{n-1}, E_{n-1}, B_{n-1}\right) \in F_{\rho, \beta, \gamma_{1}, \tau_{1}}^{l} \times E B_{\tau_{1}}^{l} \\
\left|f_{n-1}\right|_{l, \rho, \beta, \gamma_{1}, \tau_{1}} \equiv Y_{n-1} \leqq Y,
\end{gathered}
$$

with some $Y, \tau_{1}>0, \gamma_{1}>0$ and $\rho-\gamma_{1} \tau_{1} \geqq \rho / 2$, then by Lemma 2.1 and 2.6 there hold

$$
\begin{gathered}
\left(f_{n}, E_{n}, B_{n}\right) \in F_{\rho, \beta, \gamma, \tau}^{l} \times E B_{\tau}^{l}, \\
\left|E_{n}\right|_{l, \tau}+\left|B_{n}\right|_{l, \tau} \leqq 2\left(\left|E_{0}\right|_{l}+\left|B_{0}\right|_{l}\right)+a \tau Y_{n-1} \equiv G\left(\tau Y_{n-1}\right)
\end{gathered}
$$

with $a=a(\rho, \beta)$ defined in (2.5) and with some $\tau \in\left(0, \tau_{1}\right]$ and $\gamma \geqq \gamma_{1}$ satisfying

$$
\begin{gathered}
\gamma \geqq b(l) G(\tau Y) \geqq b(l)\left|B_{n}\right|_{l, \tau}(\text { or } \gamma=\gamma(c) \geqq b(l) G(\tau Y) / c), \\
\rho-\gamma \tau \geqq / 2 \quad(\text { for } c \in[1, \infty)) .
\end{gathered}
$$

By virtue of Lemma 2.6 (i) and the assumption (3.12) $)_{n-1}$ we have

$$
\begin{aligned}
\left|f_{n}\right|_{l, \rho, \beta, \gamma, \tau} & =Y_{n} \leqq e^{b \tau}\left|f_{0}\right|_{l, \rho, \beta} \leqq e^{\tau d\{G(\tau Y)+1\}}\left|f_{0}\right|_{l, \rho, \beta} \equiv F(\tau, Y), \\
b & =b(l)\left\{1+(\rho+|\beta|+1)\left|E_{n}\right|_{l, \tau}+\left|B_{n}\right|_{l-1, \tau}\right\}, \\
d & =b(l)(\rho+|\beta|+1) .
\end{aligned}
$$

Let $\widetilde{Y}_{0}>0$ be the smallest positive root of the equation

$$
Y=e^{\tau d(G(\tau Y)+1)}\left|f_{0}\right|_{l, \rho, \beta}(=F(\tau, Y))
$$

If $\tau>0$ is sufficiently small (the bound is estimated by $a=a(\rho, \beta), d,\left|E_{0}\right|_{l}+\left|B_{0}\right|_{l}$ and $\left|f_{0}\right|_{l, \rho, \beta}$ ), then Eq. (3.17) has two positive roots $0<\tilde{Y}_{0}<\tilde{Y}_{1}$. We fix such a $\tau>0$, and see easily the following,

$$
0 \leqq Y_{n-1} \leqq \tilde{Y}_{0} \text { implies } 0 \leqq Y_{n}=F\left(\tau, Y_{n-1}\right) \leqq \tilde{Y}_{0}
$$

Noting that $\left|f_{0}\right|_{l, \rho, \beta}=Y_{0}<\tilde{Y}_{0}$, we have

$$
\left|f_{n}\right|_{l, \rho, \beta, \gamma, \tau}=Y_{n}<\tilde{Y}_{0}, \quad n \geqq 1,
$$

for $\gamma>0$ and $\tau>0$ which are chosen to satisfy the additional conditions (3.14) and (3.15). 
Summing up the above arguments, we have

$$
\begin{gathered}
\left|f_{n}\right|_{l, \rho, \beta, \gamma, \tau} \leqq \widetilde{Y}_{0} \equiv Z_{0}\left|f_{0}\right|_{l, \rho, \beta}, \quad Z_{0}=e^{\tau d\left(G\left(\tau \tilde{Y}_{0}\right)+1\right)}>1, \\
\left|E_{n}\right|_{l, \tau}+\left|B_{n}\right|_{l, \tau} \leqq G\left(\tau Y_{n}\right) \leqq G\left(\tau \widetilde{Y}_{0}\right) .
\end{gathered}
$$

From the definition of $\left(f_{n}, E_{n}, B_{n}\right)$ we obtain

$$
\begin{gathered}
f_{n+1}-f_{n}=\int_{0}^{t} U\left(t, s ; E_{n+1}, B_{n+1} / c\right) k_{n}(s) d s, \\
k_{n}=-\left(E_{n+1}-E_{n}\right) \cdot \nabla_{v} f_{n}-\left(\frac{v}{c} \times\left(B_{n+1}-B_{n}\right)\right) \cdot \nabla_{v} f_{n}, \quad n \geqq 1, \\
\left(\begin{array}{c}
E_{n+1}-E_{n} \\
B_{n+1}-B_{n}
\end{array}\right)=\int_{0}^{t} e^{c(t-s) A}\left(\begin{array}{c}
G_{n}(s) \\
0
\end{array}\right) d s . \\
G_{n}(t, x)=-4 \pi \int v\left(f_{n}-f_{n-1}\right) d v,
\end{gathered}
$$

In a similar way as in (2.34) we see from (3.21) that

$$
\begin{aligned}
& \left|f_{n+1}-f_{n}\right|_{0, \rho-\gamma t, \beta-1} \leqq \int_{0}^{t} e^{r d G\left(r \tilde{Y}_{0}\right)}\left|k_{n}(r)\right|_{0, \rho-\gamma r, \beta-1} d r \\
& \quad \leqq t e^{t d G\left(t \tilde{Y}_{0}\right)} b_{2} \widetilde{Y}_{0}\left(\left|E_{n+1}-E_{n}\right|_{0, t}+\left|B_{n+1}-B_{n}\right|_{0, t}\right) .
\end{aligned}
$$

On the other hand applying Lemma 2.1 to (3.22), we obtain

$$
\left|E_{n+1}-E_{n}\right|_{0, t}+\left|B_{n+1}-B_{n}\right|_{0, t} \leqq a(\rho, \beta-1) t\left|f_{n}-f_{n-1}\right|_{0, \rho, \beta-1, \gamma, t}, \quad 0 \leqq t \leqq \tau .
$$

Combining (3.23) and (3.24), we have

$$
\left|f_{n+1}-f_{n}\right|_{0, \rho, \beta-1, \gamma, t} \leqq t^{2} e^{t d G\left(t \tilde{Y}_{0}\right)} b_{2} \tilde{Y}_{0} a(\rho, \beta-1)\left|f_{n}-f_{n-1}\right|_{0, \rho, \beta-1, \gamma, t} .
$$

If we choose $T \in(0, \tau]$ so that there holds

$$
T^{2} e^{T d G\left(T \widetilde{Y}_{0}\right)} b_{2} \widetilde{Y}_{0} a(\rho, \beta-1)<1,
$$

then $\left\{f_{n}\right\}$ is a Cauchy sequence in $C_{\gamma}^{0}\left([0, T] ; H_{\rho, \beta-1}^{0}\right)$. By $(3.12)_{n}$ and by a similar argument as in the proof of Lemma 2.6, we see that $\left\{f_{n}\right\}$ is a Cauchy sequence in $C_{\gamma}^{0}\left([0, T] ; H_{\rho, \beta-\delta}^{l-\delta}\right), 0<\delta \leqq 1$. This argument and (3.24) show that $\left\{\left(E_{n}, B_{n}\right)\right\}$ is a Cauchy sequence in $C^{0}\left([0, T] ; H^{l-\delta}\right), 0<\delta \leqq 1$. Putting

$$
\begin{aligned}
f(t) & =s-\lim f_{n}(t) \quad \text { in } H_{\rho-\gamma t, \beta-\delta}^{l-\delta}, & & 0<\delta \leqq 1, \\
(E(t), B(t)) & =s-\lim \left(E_{n}(t), B_{n}(t)\right) \quad \text { in } H^{l-\delta}, & & 0<\delta \leqq 1,
\end{aligned}
$$

We see that $f \in F_{\rho, \beta, \gamma, T}^{l-\delta},(E, B) \in E B_{T}^{l-\delta}, 0<\delta \leqq 1$, and also $(f, E, B)$ satisfies (3.1)-(3.1) and (3.2)-(3.2) 0 .

Noting that $\left\{f_{n}(t)\right\}$ is weakly pre-compact in $H_{\rho-\gamma t, \beta}^{l}$, we see

$$
f(t)=w-\lim f_{n}(t) \text { in } H_{\rho-\gamma t, \beta}^{l}, \quad 0 \leqq t \leqq T, \quad|f|_{l, \rho, \beta, \gamma, T} \leqq \widetilde{Y}_{0} \leqq Z_{0}\left|f_{0}\right|_{l, \rho, \beta} .
$$


Similarly we have

$$
\begin{aligned}
(E(t), B(t)) & =w-\lim \left(E_{n}(t), B_{n}(t)\right) \quad \text { in } H^{l}, \quad 0 \leqq t \leqq T, \\
|E|_{l, T}+|B|_{l, T} & \leqq 2\left(\left|E_{0}\right|_{l}+\left|B_{0}\right|_{l}\right)+a T \widetilde{Y}_{0}=G\left(T \tilde{Y}_{0}\right) .
\end{aligned}
$$

By the separability of the Hilbert spaces $H^{l}$ and $H_{\rho-\gamma t, \beta}^{l}$ we can show that $(E(t), B(t))$ and $f(t)$ are strongly measurable in $[0, T]$. Then, from the integral representation of the solution $(E(t), B(t))$ of $(3.1)-(3.1)_{0}$, it is proved that $(E, B) \in E B_{T}^{l}$. By virtue of Lemma 2.6, $f \in F_{\rho, \beta, \gamma, T}^{l}$, since $f(t)=U(t, 0 ; E, B / c) f_{0}$.

The uniqueness of the solution is easily proved by applying the same inequalities as (3.23) and (3.24) to the solution $\left(f_{1}, E_{1}, B_{1}\right)$ and $\left(f_{2}, E_{2}, B_{2}\right)$ of (3.1)-(3.2). Here we take $\rho=\gamma=0$ and $\beta=\beta^{\prime}$ with $a=a\left(0, \beta^{\prime}-1\right)<\infty$. No other differences occur. The last assertion of Theorem 3.1 follows from the fact that $f_{n}$ and $\left(E_{n}, B_{n}\right)$ satisfy $[F . \tilde{1}]_{\rho, \beta, \gamma, T}^{l}$ and $\left[E B . \tilde{1}^{\prime}\right]_{T}^{l}$, respectively, and the estimates (3.16) and (3.20) hold uniformly in $c \in[1, \infty)$. Thus we have completed the proof.

If the initial density $f_{0}(x, v)$ satisfies the support condition

$$
f_{0}(x, v)=0 \text { for }|v| \geqq R_{0},
$$

then we can adapt, instead of the Banach scale, simpler norms to estimate $\left(v \times \nabla_{x} B\right) \cdot \nabla_{v} f$. In fact we have

Theorem 3.2. Let $f_{0} \in H_{0, \beta}^{l}$ and satisfy the condition (3.31), and $\left(E_{0}, B_{0}\right) \in H^{l}$ with $l \geqq 3$ and $\beta>7 / 2$. Then there exists a unique solution $(f, E, B)$ of the Vlasov-Maxwell equation (3.1)-(3.1 $)_{0}$ and (3.2)-(3.2) $)_{0}$ in the time interval $[0, T]$ satisfying (3.2)-(3.6) with $\rho=\gamma=0$ and

$$
f(t, x, v)=0 \quad \text { for } \quad|v| \geqq R_{0}+t\|E\|_{0, T}, \quad 0 \leqq t \leqq T .
$$

$T, Y_{0}$ and $Z_{0}$ depend on $l, \beta,\left|f_{0}\right|_{l, 0, \beta},\left|E_{0}\right|_{l}\left|B_{0}\right|_{l}$ and $R_{0}$ but not on $c \in[1, \infty)$, and are determined by the solvability condition of $(3.39)$ with $a=a(0, \beta)$. If $\beta-m>5 / 2$ and $m \leqq l$, then

$$
(f, E, B) \in \bigcap_{j=0}^{m} M^{j}\left([1, \infty) ; C^{0}\left([0, T] ; H_{0, \beta-j}^{l-j} \times H^{l-j}\right)\right) .
$$

Moreover, if $f_{0}$ satisfies the support condition

$$
f_{0}(x, v)=0 \text { for }|x| \geqq R_{1} \quad \text { or } \quad|v| \geqq R_{0},
$$

then the solution $f(t, x, v)$ also satisfies

$$
\begin{aligned}
& f(t, x, v)=0 \quad \text { for } \quad|x| \geqq R_{1}+t R_{0}+\frac{1}{2} t^{2}\|E\|_{0, t} \quad \text { or } \\
& |v| \geqq R_{0}+t\|E\|_{0, t} .
\end{aligned}
$$

Sketch of the Proof. The support condition (3.31) and (3.33) are easy consequences of Lemma 2.2 and the definition of $U(t, s ; E, B / c) f_{0}$. Defining $\left(F_{n}, E_{n}, B_{n}\right)$ by (3.7)-(3.10), and noting that

$$
f_{n}(t, x, v)=0 \quad \text { for } \quad|v| \geqq R_{0}+t\left\|E_{n}\right\|_{0, t},
$$


we obtain by Lemma 2.6 (iii) and Lemma 2.1,

$$
\begin{gathered}
\left|f_{n}(t)\right|_{l, 0, \beta} \leqq e^{\sigma_{n} t}\left|f_{0}\right|_{l, 0, \beta}, \\
\tilde{b}_{n}=b(l)\left\{1+(|\beta|+1)\left(\left|E_{n}\right|_{l, T}+\left|B_{n}\right|_{l, T}\right)\right\}+c(l)\left|B_{n}\right|_{l, T}\left(R_{0}+t b_{2}\left|E_{n}\right|_{l, T}\right), \\
\left|E_{n}\right|_{l, t}+\left|B_{n}\right|_{0, t} \leqq 2\left(\left|E_{0}\right|_{l}+\left|B_{0}\right|_{l}\right)+a t\left|f_{n-1}\right|_{l, 0, \beta, t} \\
=G\left(t\left|f_{n-1}\right|_{l, 0, \beta, t}\right), \quad a=a(0, \beta) .
\end{gathered}
$$

If we assume

$$
\left|f_{n-1}\right|_{l, 0, \beta, \tau}=Y_{n-1} \leqq Y,
$$

then we have with $d=b(l)(|\beta|+1)+c(l)$ and $R_{1}=\max \left\{1, R_{0}\right\}$,

$$
\left|f_{n}\right|_{l, 0, \beta, \tau}=Y_{n} \leqq e^{\tau d\left\{1+G\left(\tau Y_{n-1}\right)\right\}\left\{R_{1}+\tau b_{2} G\left(\tau Y_{n-1}\right)\right\}}\left|f_{0}\right|_{l, 0, \beta} .
$$

If we choose $T>0$ so small that the equation

$$
Y=e^{T d\{1+G(T Y)\}\left\{R_{1}+T b_{2} G(T Y)\right\}}\left|f_{0}\right|_{l, 0, \beta}
$$

has two positive roots $0<\tilde{Y}_{0}<\tilde{Y}_{1}$, then we can conclude that

$$
0<Y_{n-1} \leqq \widetilde{Y}_{0} \text { implies } 0<Y_{n} \leqq \widetilde{Y}_{0} \text {. }
$$

Since $\left|f_{0}\right|_{l, 0, \beta}=Y_{0}<\tilde{Y}_{0}$, we have

$$
\left|f_{n}\right|_{l, 0, \beta, T}=Y_{n}<\tilde{Y}_{0} .
$$

The rest of the proof is quite similar to the proof of Theorem 3.1.

Remark. The latter part of Theorem 3.2 was first proved by Wollmann [9].

Acknowledgement. The author appreciates the kind advice of Prof. Ukai. Lemma 2.4 was introduced by his suggestion, and the proof of Lemma 2.5 was much clarified by virtue of this lemma.

\section{References}

1. Arsen'ev, A. A.: Local uniqueness and existence of a classical solution of Vlasov system of equation. Sov. Math., Dokl. 15, 1223-1225 (1974)

2. Bardos, C., Degond, P.: Global existence for the Vlasov-Poisson Equation in 3 space variables with small initial data. Ann. Inst. Henri Poincaré-Analyse non linéaire 2, 101-118 (1985)

3. Batt, J.: Global symmetric solutions of the initial value problem of steller dynamics. J. Differ. Equations 25, 342-364 (1977)

4. Cooper. J., Klimas, A.: Boundary value problems for the Vlasov-Maxwell equation in one dimension. J. Math. Anal. Appl. 75, 306-329 (1980)

5. Duniec, J.: On an initial value problem for nonlinear systems of Vlasov-Maxwell equations. Bull. Acad. Plar. Sci. Ser. Sci. Tech. 21, 97-102 (1973)

6. Iordanskii, S. V.: The Cauchy problem for the kinetic equation of plasma. Mat. Inst. Steklova 60, 181-194 (1961) English transl. Transl. Am. Math. Soc. (2) 35, 351-363 (1964)

7. Neunzert, H., Petry, K. H.: Ein Existenzsatz fur die Vlasov-Gleichung mit selbstkonsistentem Magnetfeld. Math. Mech. Appl. Sci. 2, 429-444 (1980)

8. Ukai, S., Okabe, T.: On classical solutions in the large in time of two dimensional Vlasov's equation. Osaka J. Math. 15, 245-261 (1978)

9. Wollmann, S.: An existence and uniqueness theorem for the Vlasov-Maxwell system. Commun. Pure Appl. Math. 37, 457-462 (1984) 
10. Degond, P.: Local existence of solutions of the Vlasov-Maxwell equations and convergence to the Vlasov-Poisson for infinite light velocity (1984), Preprint

11. Asano, K. Ukai, S.: On the Vlasov-Poisson limit of the Vlasov-Maxwell equations (1985), to appear in: Qualitative analysis of nonlinear differential equations. Studies in Math. its Appl., North-Holland

Communicated by H. Araki

Received November 2, 1984; in revised form February 17, 1986 